\title{
Treino de competências académicas e pre-académicas em crianças com
}

problemas escolares

\section{Training of academic and pre-academic competences in children with school problems}

\author{
Edgar Galindo, Helena Coradinho \\ Universidade de Évora, Portugal
}

\begin{abstract}
Resumo
O objetivo principal deste artigo é apresentar os resultados obtidos na aplicação de procedimentos de treino baseados nas técnicas da Análise Comportamental Aplicada (ACA) a alunos na situação de insucesso escolar. Programas de treino anteriormente aplicados com sucesso a alunos do $1 .^{\circ}$ ao $3 .^{\circ}$ ano de escolaridade, foram aplicados nesta investigação a três alunos com problemas de insucesso escolar do $4 .^{\circ}$ ano de escolaridade. Foram aplicados como instrumentos para medir o desempenho dos alunos o Subteste de Aritmética da WISC-III e as Provas de Avaliação do Método Distema, incluido a prova de Despsite de Dislexia, antes e após a intervenção com técnicas da ACA. Os resultados obtidos mostraram ganhos advancers nas competências de compreensão verbal, leitura, ortografia e Matemática em todos os alunos. Todas as formas de avaliação aplicadas mostraram os mesmos resultados. Foram, assim, replicados os resultados obtidos noutros estudos similares realizados em Portugal. Concluiu-se assim que, uma intervenção de caráter individual baseada nas técnicas da ACA parece melhorar o desempenho escolar de alunos do ensino básico na situação de insucesso escolar.
\end{abstract}

Palavras-chave: desempenho escolar, school failure, Análise Comportamental Aplicada.

\footnotetext{
Abstract

The aim of this paper is to present the results of an intervention program based in Applied Behavior Analysis (ABA) to train academic skills in children with school failure problems. In the current research, training programs successfully applied to 1st and 2nd school year children were used to train three children attending the 4th school year. An evaluation before and after the intervention with ABA techniques was carried on using WISC-III Arithmetics Sub-test and tests of the Distema Method, including a Dyslexia identification test. Results showed advances in Verbal Comprehension, Orthography and Arithmetics in all children. All applied evaluation tests showed similar results. Results obtained in Portugal with younger children were replicated. As a conclusion, it is stressed thar intervention programs based on $\mathrm{ABA}$ seem to increase academic performance of children with school failure problems.

Keywords: school achievement, school failures, Applied Behavior Analysis
}

\section{Introdução}

O insucesso escolar é hoje o problema de maior gravidade do sistema de ensino português (Bastos, Fernandes \& Passos, 2009). Definir o insucesso escolar tem-se mostrado uma tarefa complexa (Marchesi \& Pérez, 2003), porém considera-se que um aluno apresenta problemas de insucesso escolar quando, de acordo com os critérios do professor e da escola, manifesta um desempenho escolar abaixo da média para a sua faixa etária e ciclo escolar (Cort, 2008). Em termos práticos, considera-se que um aluno se encontra na situação de insucesso escolar quando de acordo com os critérios do professor é referenciado por qualquer tipo de problemas de insucesso escolar, como é o caso de anteriores retenções ou a probabilidade de vir a ficar retido (Galindo, 2015).

Hoje em dia, acredita-se que não é possível explicar o insucesso escolar através de um único fator (Marchesi \& Pérez, 2003). Existe, portanto, um vasto leque de fatores que contribuem para o insucesso escolar, sendo ele um fenómeno multidimensional, ou seja, com múltiplas causas (Marchesi \& Pérez, 2003; Moreira, Dias, Vaz \& Vaz, 2013). Sem menosprezar a importância de cada um destes fatores, neste estudo será dado enfoque à influência dos fatores individuais, especialmente, dos aspetos motivacionais e das competências prévias do aluno no desempenho escolar.

De entre os inúmeros fatores que têm impacto no insucesso escolar, vários são os investigadores que colocam em lugar de destaque a motivação (Greene, Pasnak \& Romero, 2009), e os conhecimentos prévios dos alunos no seu desempenho escolar (Hailikari, Nevgi \& Lindblom-Ylänne, 2007; Marchesi \& Pérez, 2003; e Yenilmez, Sungur \& Tekkaya, 2006). Uma série de investigadores identificaram, inclusive, um conjunto diversificado de competências que são pré-requisito para a aprendizagem de outras habilidades, como a leitura, a escrita e a aritmética, especialmente, nos anos iniciais de escolaridade, pelo que quando um aluno não as apresenta toda a aprendizagem futura será condicionada, podendo culminar no insucesso escolar (Leppänen, Niemi, Aunola 
\& Nurmi, 2004; Levingston, Neef \& Cihon, 2009; Wood, Frank \& Wacker, 1998). Doutra parte, na literatura da área, está comprovado que os métodos de ensino individualizados são o método mais eficaz de instrução (Siler \& VanLehn, 201, Lake, Davis \& Madden, 2011). Existem, inclusive, claras evidências de que os métodos de ensino individualizados são uma das estratégias pedagógicas mais eficazes em casos de insucesso escolar (Marchesi \& Pérez, 2003; Wasik \& Slavin, 1990). Existe também um corpo crescente de pesquisas que têm dado ênfase ao papel de intervenções de cariz individualizado levadas a cabo por figuras-chave na vida dos alunos, como por exemplos os pais, comprovando-se que estes podem ser um elemento viável e eficaz na implementação de uma intervenção que recorre a métodos de ensino individualizados (Preece, 2014). É com base nisto, que a presente investigação oferece um conjunto diversificado de programas de treino elaborados especificamente, de forma a serem implementados por outros elementos envolvidos no processo de ensino-aprendizagem, que não o profesor

Outro elemento importante é que essa intervenção individualizada deve ser implementada o mais precocemente possível, daí a necessidade de intervenção logo no $1 .^{\circ}$ ciclo do ensino básico ou até antes se possível. Hailikari et al. (2007), Marchesi e Pérez (2003), e Yenilmez et al. (2006), justificam a necessidade de uma intervenção precoce, pelo facto de certos conhecimentos funcionarem como pré-requisito para a aquisição de outros.

Neste contexto, é importante propor estratégias de combate ao insucesso escolar complementares às muitas já existentes (Marchesi \& Pérez, 2003). Contributo esse assente na disponibilização de uma intervenção precoce e individualizada, administrada de acordo com os princípios da Análise Comportamental Aplicada (ACA).

A partir do conhecimento acumulado, foi elaborada uma estratégia de intervenção baseada nos princípios e métodos cognitivos e comportamentais, regida pelas regras da ACA (Kazdin, 2013; Martin \& Pear, 2009; Miltenberger, 2012; Thomas, 2009). De acordo com Galindo (2015), ao longo dos anos, tem havido uma preocupação crescente por parte da psicologia cognitivocomportamental não só para diagnosticar, mas também para resolver problemas, a qual levou ao desenvolvimento de um conjunto alargado de técnicas de diagnóstico e reabilitação destinadas à resolução de uma série de problemas psicológicos e ao treino de um conjunto abrangente de competências. Tal situação levou a que, hoje em dia, exista um corpo alargado de investigadores que provou que as técnicas cognitivocomportamentais são uma ferramenta útil e eficaz para lidar com uma série de problemas, entre os quais, as perturbações do desenvolvimento inteletual, as perturbações do espetro do autismo, as perturbações visuais, as perturbações auditivas, as perturbações alimentares, a paralisia cerebral, entre muitas outras (Miltenberger, 2012).

A ACA diz respeito a um processo no qual um determinado comportamento é modificado através da aplicação sistemática de princípios e técnicas derivados da teoria da aprendizagem e da Psicologia experimental
(Kazdin, 2013; e Miltenberger, 2012). A ACA se preocupa com a análise do comportamento, na medida em que identifica a relação existente entre os estímulos ambientais e o comportamento humano, tentando compreender as razões para a ocorrência do mesmo e, também com a sua modificação, já que envolve o desenvolvimento e implementação de procedimentos com o objetivo de alterar/modificar um determinado comportamento socialmente significativo (através da modificação dos estímulos ambientais que influenciam esse comportamento) e assim, melhorar algum aspeto da vida do indivíduo (Miltenberger, 2012).

Para Galindo (2015) uma intervenção baseada na ACA começa com um diagnóstico comportamental, que em casos de insucesso escolar envolve três partes: a realização de uma entrevista ao professor e aos os pais do aluno, a análise dos relatórios existentes e/ou processos pedagógicos e a aplicação de provas comportamentais que permitem avaliar três áreas (área da autossuficiência básica, área académica e área do comportamento social); após a avaliação comportamental, é de extrema importância definir uma estratégia de intervenção, a partir da qual deverão ser construídos os programas de treino destinados a resolver os problemas apresentados pelo aluno. Programas esses que devem ser concebidos especificamente para cada aluno, ou seja, de acordo com as suas necessidades e caraterísticas e ainda com base nos resultados obtidos na avaliação comportamental, sendo necessária a construção de um programa de treino para cada comportamento considerado deficitário. Deste modo, seja qual for o programa de treino deverá ser constituído pelos seguintes aspetos: objetivo geral, objetivos específicos, definição do comportamento- alvo, definição do repertório de entrada, ou seja, dos prérequisitos, fases, procedimentos, materiais, local do treino, formas de registo, processos de avaliação e ainda a especificação dos aspetos motivacionais (Galindo, 2015).A par disto, os aspetos motivacionais também são de extrema importância, já que têm um papel muito importante no sucesso do programa de treino, sendo por isso fundamental recorrer à aplicação de um sistema de economia de fichas para melhorar a motivação dos alunos alvo de intervenção. (Kazdin, 1982; Martin \& Pear, 2009).

Galindo (2015) aplicou estes programas a crianças com problemas de insucesso escolar nos primeiros três anos do ensino básico; observou que todos os alunos acompanhados progrediram e adquiriram os repertórios ensinados, independentemente da causa do problema, da idade, do tempo de treino ou da competência treinada. A maioria das crianças adquiriu também uma maior segurança no seu desempenho, melhorou 0 comportamento social e, consequentemente, a relação com o professor e a integração na escola.

O principal objetivo deste artigo é alargar os resultados obtidos na aplicação de procedimentos baseados na ACA, destinados a ajudar a eliminar os problemas de insucesso escolar. Neste caso em concreto, programas de treino de competências académicas e pré-académicas foram aplicados a um novo ano de escolaridade ( $4 .^{\circ}$ ano de escolaridade). 


\section{Método}

\section{Participantes}

Participaram neste estudo 3 alunos do $4 .^{\circ}$ ano de escolaridade, aqui designados por $\mathrm{A}, \mathrm{B}$ e $\mathrm{C}$ com idades compreendidas entre os 10 e os 11 anos pertencentes uma escola do Alentejo, referidos pelo professor por problemas de insucesso escolar, e que cumpriram os critérios de inclusão e exclusão definidos. A segunda autora funcionou como uma tutora e, foi responsável pela avaliação dos alunos e implementação dos programas de treino.

\section{Instrumentos}

Os instrumentos utilizados para a avaliação foram os seguintes:

1. Subteste de Aritmética da WISC-III (SA).

2. Provas de Avaliação do Método Distema: Avaliação da Consciência Silábica e Fonémica (PCSF), Ortografia (PO), Velocidade Leitora (PL) e ainda a Prova de Despiste de Dislexia (PDD).

3. Questionários. Três questionários constituídos por Compreensão Verbal, Leitura, Ortografia e Matemática.

4. Provas de Diagnóstico Comportamental do Insucesso Escolar de Galindo (2015) ${ }^{l}$ : Imitação Vocal, Articulação, Compreensão Verbal, Leitura de Letras, Palavras e Frases, Ditado (Letras, Palavras, Frases e Combinações Problemáticas), Língua Portuguesa do $3 .^{\circ}$ ano, e Matemática do $2 .^{\circ}$ ano e do $3 .^{\circ}$ ano.

5. Entrevistas. Semi-estruturadas com a duração de cerca de 20 minutos e de acordo com um guião com 6 blocos temáticos.

Já os instrumentos utilizados durante a fase de treino foram os seguintes:

6. Programas de Treino. Foram concebidos quatro programas de treino (Programa de Treino para a Compreensão Verbal, para a Leitura, para a Ortografia e para a Matemática de $4 .^{\circ}$ ano), com base nos programas de treino de Galindo (2015).

\section{Procedimentos}

De início, procedeu-se à avaliação inicial dos alunos $\mathrm{A}$, $\mathrm{B}$ e C, com recurso aos instrumentos de avaliação apresentados anteriormente. Posteriormente, foram definidos os objetivos de intervenção para cada um dos alunos, construídos e implementados os programas de treino que serviram de base à intervenção. Ao longo da intervenção foram realizadas duas sessões semanais com a duração de hora e meia com cada um dos alunos estudados, após o período normal de aulas. Todas as sessões decorreram num pequeno gabinete da escola e foram levadas a cabo pela tutora. Finalizada a intervenção todos os alunos foram alvo de uma avaliação final. Nesta mesma avaliação final foram utilizados os mesmos instrumentos que na avaliação inicial. Após a intervenção, foi realizada uma entrevista aos alunos, e aplicados questionários ao professor titular da turma, com o objetivo de conhecer a sua opinião face à intervenção.
Aliado a isto, no decorrer da intervenção todos os alunos foram avaliados constantemente, de forma a poder-se monitorizar a mesma e verificar a progressão dos mesmos.

\section{Resultados}

A figura 1 mostra os resultados obtidos pela aluna $\mathrm{A}$. No pré-teste, obteve uma pontuação direta de 13 no SA. Já na PCSF apresentou 4 respostas incorretas ao nível da tarefa de Segmentação Silábica (SS), 5 na tarefa de Identificação Fonémica (IF), 2 na tarefa de Segmentação Fonémica (SF) e 1 na tarefa Completar Palavras (CP). Por sua vez, na PDD foram detetados 14 erros na leitura e na PL foram contabilizadas 84 palavras lidas corretamente durante 1 minuto. Por último, foram detetados 45 erros ortográficos na PO. No pós-teste, a aluna A alcançou uma pontuação direta de 16 no SA. Enquanto, na PCSF exibiu 1 resposta incorreta na tarefa SS, 1 na tarefa IF, 0 na tarefa SF, 0 na tarefa CP. Já na PDD foram cometidos 10 erros na leitura e na PL foram lidas 136 palavras de forma correta num minuto. Por fim, na PO foram cometidos 37 errros ortográficos.

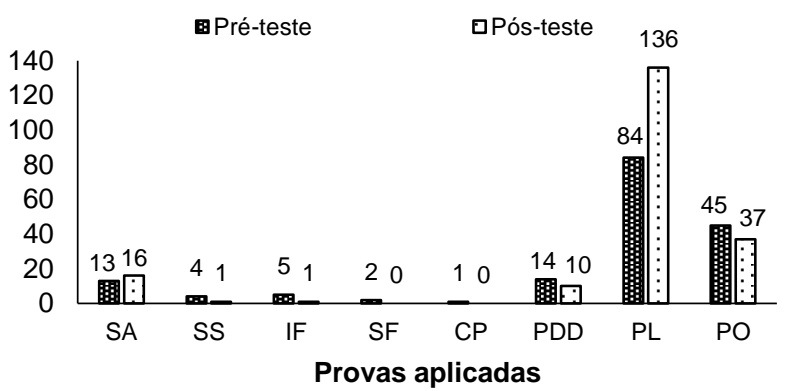

Figura 1. Resultados obtidos pela aluna A no pré e pósteste por prova aplicada.

A figura 2 apresenta os resultados obtidos pelo aluno B. No pré-teste, apresentou 3 respostas incorretas na tarefa SS, 16 na tarefa IF, 6 na tarefa SF e 6 na tarefa CP da PCSF. Na PDD foram cometidos 22 erros na leitura, enquanto na PL foram lidas corretamente 68 palavras num minuto. Na PO foram detetados 75 erros ortográficos. No pós-teste, o aluno B exibiu 2 respostas incorretas na tarefa SS, 10 na tarefa IF, 1 na tarefa SF e 0 na tarefa $\mathrm{CP}$ da $\mathrm{PCSF}$. No que diz respeito, à $\mathrm{PDD}$ não foi cometido nenhum erro na leitura. Já na PL foram registadas 126 palavras lidas corretamente num minuto e na PO foram cometidos 64 erros ortográficos.

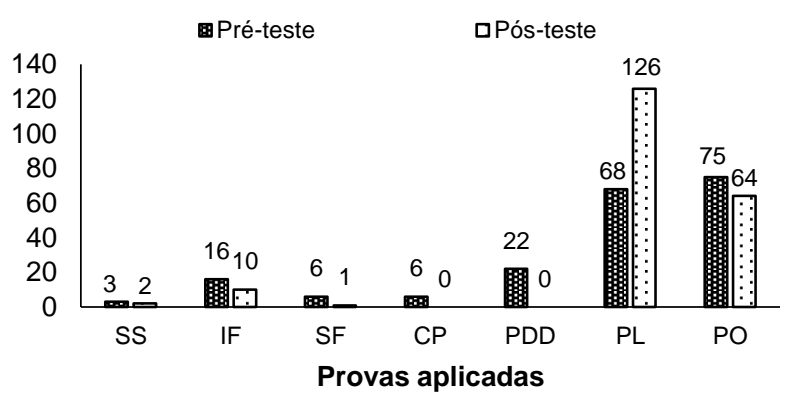

${ }^{1}$ Os resultados destas provas serão objeto de outro artigo. 
Figura 2. Resultados obtidos pelo aluno B no pré e pósteste por prova aplicada.

A figura 3 apresenta os resultados obtidos pelo aluno C. No pré-teste, exibiu 3 respostas incorretas na tarefa SS, 7 na tarefa IF, 6 na tarefa SF e 1 na tarefa CP da PCSF. Na PDD apresentou 30 erros na leitura. Por outro lado, foram contabilizadas 66 palavras lidas corretamente num minuto na PL e 81 erros ortográficos na PO. No pósteste, 0 respostas incorretas nas tarefas SS, IF, SF e CP da PCSF, respetivamente. Já na PDD exibiu 7 erros na leitura e na PL foram lidas 127 palavras de forma correta num minuto. Por último, na PO foram detetados 64 erros ortográficos.

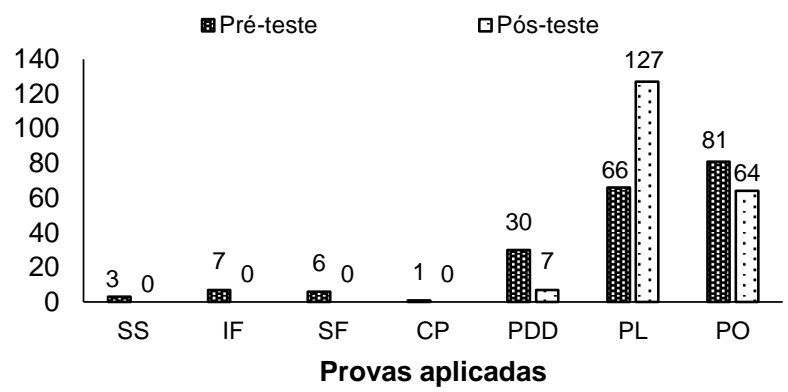

Figura 3. Resultados obtidos pelo aluno C no pré e pósteste por prova aplicada.

Quanto aos questionários, a opinião do professor titular dos alunos foi inequívoca ao concordar com o facto de ter existido uma melhoria na maioria dos indicadores relativos às competências de compreensão verbal, leitura, ortografia e matemática em todos os alunos. O professor apenas discordou com o facto de ter existido uma diminuição dos incentivos à leitura nos alunos B e C.

Os alunos destacaram os seguintes aspetos positivos: qualidade das atividades ("divertidas"), opinião pessoal da intervenção ("gostei de tudo") e qualidade da tutora ("trabalhou bem"). Por outro lado, foram apontados alguns aspetos negativos relacionados com a tutora ("falava alto"), as próprias atividades ("chatas") e a calendarização da intervenção ("poucas sessões). Foram ainda assinaladas algumas dificuldades pelos alunos, nomeadamente ao nível das atividades ("complicadas", "confusas"), da tutora ("não a ouvia bem") e do próprio aluno (nervosismo). Apesar das dificuldades relatadas, foram notadas alterações decorrentes da intervenção por todos os alunos e foram largamente citados os seus benefícios. Ao sistema de economia de fichas foram atribuídas várias vantagens por todos os alunos, que o consideraram benéfico em termos motivacionais.

\section{Discussão}

Todos os três alunos que participaram nesta investigação responderam de forma semelhante aos programas de treino implementados, já que se registou um aumento da pontuação direta no SA, uma diminuição do número de respostas incorretas em todas as tarefas da PCSF, uma diminuição do número de erros na leitura na PDD, um aumento do número de palavras lidas corretamente durante 1 minuto na PL e ainda uma diminuição do número de erros ortográficos na $\mathrm{PO}$ em todos os alunos. Tal, parece assim, sugerir que os alunos apresentaram melhorias em todas as competências treinadas, verificando-se que uma intervenção de índole cognitivo-comportamental parece melhorar o desempenho escolar de alunos do ensino básico na situação de insucesso escolar. O facto de os programas de treino implementados terem sido associados a ganhos consideráveis nas competências de compreensão verbal, leitura, ortografia e Matemática em todos os alunos não foi de todo surpreendente. Já há alguns anos que as técnicas cognitivo-comportamentais são utilizadas com eficácia no campo da educação, com vários propósitos, entre os quais, o de melhorar o desempenho escolar dos alunos, especialmente daqueles com algum tipo de dificuldades, através do treino das habilidades académicas em défice (Galindo, 2015, Jerome, Frantino, \& Sturmey, 2007; Kazdin, 2013; e Wood et al., 1998).

A apresentação de resultados semelhantes entre os três alunos que participaram nesta investigação, parece ainda mostrar a replicação dos efeitos da intervenção entre alunos, o que permite pressupor que outros alunos com as mesmas caraterísticas na situação de insucesso escolar também poderiam vir a mostrar os mesmos efeitos, ou seja, a melhorar o seu desempenho escolar com a implementação dos mesmos programas de treino.

Coletivamente, os resultados alcançados por todos os alunos coincidem em todas as provas aplicadas. Pode concluir-se que houve aprendizagem durante a fase de treino, ou seja, durante a implementação dos programas de treino.

O professor concordou com o facto de terem existido melhorias em todas as competências que foram alvo de treino em todos os alunos. Já os alunos não só relataram que apreciaram todos os programas de treino, apelidando-os de "divertidos", "cómicos" e "fixes", como também atribuíram as melhorias nas competências alvo de treino diretamente às estratégias de treino utilizadas nos programas de treino.

A utilização do sistema de economia de fichas assumiu um papel muito importante em termos motivacionais durante toda a intervenção. Levando isto em linha de conta, supõe-se que o sistema de economia de fichas foi eficaz na melhoria da motivação dos alunos, o que vai de encontro ao descrito na literatura, nomeadamente, que este tipo de procedimento é eficaz no aumento da motivação dos alunos (Kazdin, 1982; e Martin \& Pear, 2009).

Os programas de treino foram implementados por uma tutora, sem quaisquer conhecimentos em ensino/educação, o que faz notar que a implementação deste tipo de programas de treino parece ser bemsucedida quando realizada por outras pessoas que não professores. De acordo com Preece (2014) e Slavin et al. (2011) os paraprofissionais, voluntários, figuras-chave na vida dos alunos (como por exemplo os pais ou outros familiares) são elementos viáveis na implementação de intervenções que recorrem a métodos de ensino individualizados, o que vai de encontro aos resultados desta investigação.

É ainda importante referir que cada um dos programas de treino foi implementado individualmente e, que foram 
construídos de acordo com as caraterísticas e necessidades dos alunos, constituindo assim métodos de ensino individualizados. Métodos estes que, que consoante os resultados desta investigação, parecem ter sido adequados, o que é consistente com o descrito na literatura por Grisay (2003) e Wasik \& Slavin (1990).

\section{Conclusões}

Os resultados parecem demonstrar que uma intervenção de caráter individual baseada nas técnicas cognitivo-comportamentais melhora o desempenho escolar de alunos do ensino básico na situação de insucesso escolar, já que replicam os resultados obtidos por Galindo (2015) com alunos do $1 .^{\circ}$ ao $3 .^{\circ}$ ano de escolaridade e concordam com os autores citados.

Tais resultados fornecem assim um contributo importante ao nível do combate ao insucesso escolar, já que é aqui disponibilizado um conjunto de programas de treino que poderá ser aplicado a outros alunos com caraterísticas semelhantes. Na certeza de que muitas medidas e estratégias de combate ao insucesso escolar ainda estão por encontrar, só um esforço conjunto irá permitir encontrá-las, para o qual se deixa este despretensioso contributo. No entanto, espera-se que seja uma mais-valia para que sejam acrescidas as oportunidades dos alunos na situação de insucesso escolar.

\section{Referências}

Bastos, A., Fernandes, G., \& Passos, J. (2009). Analysis of school failure based on Portuguese micro data. Applied Economics Letters, 16(16), 1639-1643. http://dx.doi.org/10.1080/13504850701604094

Cort, K. (2008). Working with school failure. In H. Coleman \& C. Yeh (Eds.). Handbook of School Counseling (pp. 635-650). Philadelphia: Taylor \& Francis.

Galindo, E. (2015). O tratamento do insucesso escolar com técnicas da psicología: Manual prático. Lisboa: Livros Horizonte.

Grisay (2003). Repetir curso o adecuar el curriculum. In A. Marchesi \& C. Gil (Coords.). El Fracaso Escolar: Una Perspectiva Internacional (pp. 101-127). Madrid: Alianza Editorial.

Greene, M., Pasnak, R., \& Romero, S. (2009). A Time Lag Analysis of Temporal Relations Between Motivation, Academic Achievement, and Two Cognitive Abilities. Early Education and Development, 20(5), 799-825. http://dx.doi.org/10.1080/10409280802581177

Hailikari, T., Nevgi, A., \& Lindblom- Ylänne, S. (2007). Exploring alternative ways of assessing priorknowledge, its components and their relation to student achievement: a mathematics based case study. Studies in Educational Evaluation, 33, 320-337. http://dx.doi.org/10.1016/j.stueduc.2007.07.007

Jerome, J., Frantino, E., \& Sturmey, P. (2007). The effects of errorless learning and backward chaining on the acquisition of internet skills in adults with developmental disabilities. Journal of Applied
Behavior Analysis, 40, 185-189. http://dx.doi.org/10.1901/jaba.2007.41-06

Kazdin, A. (1982). The token economy: A decade later. Journal of Applied Behavior Analysis, 15(3), 431-445. http://dx.doi.org/10.1901/jaba.1982.15-431

Kazdin, A. (2013). Behavior modification in applied settings. EUA: Waveland Press.

Leppänen, U., Niemi, P., Aunola, K., \& Nurmi, J. (2004). Development of reading skills among preschool and primary school pupils. Reading Research Quaterly, 39(1), 72-93. http://dx.doi.org/10.1598/RRQ.39.1.5

Levingston, H., Neef, N., \& Cihon, T. (2009). The effects of teaching precurrent behaviors on children's solution of multiplication and division word problems. Journal of Applied Behavior Analysis, 42, 361-367. http://dx.doi.org/10.1901/jaba.2009.42-361

Marchesi, A., \& Pérez, E. (2003). La comprensión del fracaso escolar. In A. Marchesi \& C. Gil (Coords.). El Fracaso Escolar: Una Perspectiva Internacional (pp. 26-50). Madrid: Alianza Editorial.

Martin, G., \& Pear, J. (2009). Modificação de comportamento: $O$ que é e como fazer. Universidade de Manitoba: ROCA.

Miltenberger, R. (2012). Behavior modification: Principles and Procedures. EUA: Cengage Learning.

Moreira, P., Dias, P., Vaz, F., \& Vaz, J. (2013). Predictors of academic performance and school engagement - Integrating persistence, motivation and study skills perspectives using person-centered and variable-centered approaches. Learning and Individual Differences, $\quad 24, \quad 117-125$. http://dx.doi.org/10.1016/j.lindif.2012.10.016

Preece, D. (2014). Providing training in positive behavioural support and physical interventions for parents of children with autism and related behavioural difficulties. Support for Learning, 29(2), 136-153. http://dx.doi.org/10.1111/1467-9604.12053

Siler, S., \& VanLehn, K. (2015). Investigating Microadaptation in One-to-One Human Tutoring. The Journal of Experimental Education, 83(3), 344-367. http://dx.doi.org/10.1080/00220973.2014.907224

Slavin, R., Lake, C., Davis, S., \& Madden, N. (2011). Effective programs for struggling readers: A bestevidence synthesis. Educational Research Review, 6, 1-26. http://dx.doi.org/10.1016/j.edurev.2010.07.002

Thomas, E. (2009). Behavior modification procedure: A sourcebook. EUA: Transaction Publishers.

Wasik, B., \& Slavin, R. (1990). Preventing early reading failure with one-to-one tutoring: a best-evidence synthesis. Washington: Office of Educational Research and http://files.eric.ed.gov/fulltext/ED324122.pdf

Wood, D., Frank, A., \& Wacker, D. (1998). Teaching multiplication facts to students with learning disabilities. Journal of Applied Behavior Analysis, 31, 323-338. http://dx.doi.org/10.1901/jaba.1998.31-323

Yenilmez, A., Sungur, S., \& Tekkaya, C. (2006). Students' achievement in relation to reasoning ability, prior knowledge and gender. Research in Science \& Technological Education, 24(1), 129-138. http://dx.doi.org/10.1080/02635140500485498 